NAKAGAWA, H., AND I. YOKOTE

KODAI MATH. SEM. REP.

24 (1972), 471-481

\title{
ON HYPERSURFACES WITH CONSTANT SCALAR CURVATURE IN A RIEMANNIAN MANIFOLD OF CONSTANT CURVATURE
}

\author{
By Hisao NaKagawa and Ichiro Yokote
}

\section{Introduction.}

It is well known that Liebmann [7] and Süss [15] proved that a compact convex hypersurface with constant mean curvature in a Euclidean space is a sphere. The tool used in the proof of this theorem is an integral formula containing the first mean curvature of the hypersurface. The position vector and the support function play an important role in the proof. In his paper [5], Hsiung has established an integral formula expressing the relation between the $j$-th mean curvature and the $(j+1)$-st mean curvature of a compact hypersurface in a Euclidean space, which is a generalization of that of Süss. By applying the integral formula, Hsiung proved a theorem which gives a sufficient condition for a compact hypersurface in a Euclidean space to be a sphere. This generalizes the theorem of Liebmann and Süss. The study in this line has been carried out by Amur [2], Reilly [10], Shahin [13], Yano and Tani [17] and others.

On the other hand, Simons [14] has recently done an important and suggestive contribution to the study of minimal submanifolds in a Riemannian manifold, in which he has given a formula for the Laplacian of the square of the norm of the second fundamental form of the submanifold. Under the stimulus of the Simons' study, Carmo, do Chern and Kobayashi [3], and Nomizu and Smyth [9], using the similar formula to that of Simons, have obtained some theorems on a compact minimal submanifold or a complete hypersurface with constant mean curvature in a Riemannian manifold of constant curvature.

The purpose of this paper is to generalize, by applying a formula of Simons' type to a compact hypersurface with constant scalar curvature in a Riemannain manifold of constant curvature, the theorem of Liebmann and Süss from the different point of view. We prove that a compact hypersurface of non-negative curvature and with constant scalar curvature in a Euclidean space is a sphere.

In $\S 1$, we state preliminaries and in $\S 2$ we obtain the main theorem (Theorem 2.3) stating a compact hypersurface $M$ with constant scalar curvature in a simply connected space form $M^{n+1}(c)$ is totally umbilic or has exactly two distinct and constant principal curvatures under suitable conditions. Making use of this pro-

Received October 7, 1971. 
perty, we prove some theorems for compact hypersurfaces in $M^{n+1}(c)$. In $\S 3$, we treat with compact convex hypersurfaces in a Euclidean space.

\section{Preliminaries.}

Let $\bar{M}$ be an $(n+1)$-dimensional Riemannian manifold of constant curvature $c$. Let $M$ be an $n(\geqq 2)$-dimensional connected Riemannian manifold and let $\phi$ : $M \rightarrow \bar{M}$ be an isometric immersion of $M$ into $\bar{M}$. As far as we are concerned with the local calculation, we may regard $\phi$ as an isometric imbedding. For simplicity, we say that $M$ is a hypersurface immersed in $\bar{M}$, and we identify a point $p$ of $M$ with the point $\phi(p)$ of $\bar{M}$. Throughout the paper, the differentiability classes of manifolds, immersions and tensor fields are assumed to be of class $C^{\infty}$.

By $g_{j i}, R_{k j i}{ }^{h}$ and $H_{j i}$ we denote components of the Riemannian metric tensor, the Riemannian curvature tensor and the second fundamental tensor of $M$, respectively. Then the equation of Gauss for the hypersurface $M$ is given by

$$
R_{k j i h}=c\left(g_{k h} g_{j i}-g_{k i} g_{j h}\right)+H_{k h} H_{j i}-H_{k i} H_{j h},
$$

and that of Codazzi by

$$
\nabla_{k} H_{j i}-\nabla_{j} H_{k \imath}=0 \text {, }
$$

where $\nabla$ denotes the operator of covariant differentiation with respect to the induced Riemannian connection of $M$. We define the function $f$ on $M$ by

$$
f=H_{j i} g^{i i}=H_{j}{ }^{3},
$$

which is globally defined on $M$ up to the sign. Transvecting equation (1.1) with $g^{k h}$, we get

$$
R_{j i}=c(n-1) g_{j i}+f H_{j i}-H_{j r} H_{i}^{r},
$$

where $R_{j i}$ are components of the Ricci tensor. We denote by $R$ the scalar curvature of $M$. That is to say, we put

$$
R=R_{j i} g^{j i} .
$$

Transvecting equation (1.4) with $g^{j i}$ and remembering the definition (1.3) of $f$, we obtain

$$
R=c n(n-1)+f^{2}-H_{j i} H^{j i} .
$$

Now, applying the Ricci identity to $H_{j i}$ and taking account of equation (1.2) of Codazzi, we have

$$
\nabla_{j} \nabla_{\imath} f=\nabla_{j} \nabla_{i} H_{k}{ }^{k}=\nabla^{k} \nabla_{k} H_{j i}-R_{\jmath r} H_{i}{ }^{r}-R_{j k \imath}{ }^{r} H_{r}{ }^{k},
$$

where $\nabla^{k}=g^{k i} \nabla_{\imath}$. Substituting (1.1) and (1.2) into the relation above, we obtain 


$$
\Delta H_{j i}=\nabla_{j} \nabla_{\imath} f+c\left(n H_{j i}-f g_{j i}\right)+f H_{j r} H_{i}{ }^{r}-H_{r s} H^{r s} H_{j i},
$$

where $\Delta=\nabla_{r} \nabla^{r}$ is the differential operator of Laplace and Beltrami. This leads to the equation

$$
H^{j i} \Delta H_{j i}=H^{j i} \nabla_{j} \nabla_{\imath} f+c\left(n H_{j i} H^{j i}-f^{2}\right)+f H_{\jmath r} H_{i}^{r} H^{j i}-\left(H_{j i} H^{j i}\right)^{2},
$$

which is due to Chern, do Carmo and Kobayashi [3]. Making use of the principal curvatures $k_{1}, k_{2}, \cdots, k_{n}$ of $M$, we get

$$
c\left(n H_{j i} H^{j i}-f^{2}\right)+f H_{j r} H_{\imath}^{r} H^{j i}-\left(H_{j i} H^{j i}\right)^{2}=\sum_{i<j}\left(c+k_{i} k_{j}\right)\left(k_{i}-k_{j}\right)^{2} .
$$

This implies that

$$
H^{j i} \Delta H_{j i}=H^{j i} \nabla_{j} \nabla_{\imath} f+\sum_{i<j}\left(c+k_{i} k_{j}\right)\left(k_{i}-k_{j}\right)^{2} .
$$

The equation (1.6) has been obtained by Nomizu and Smyth [9]. By a simple computation, equation (1.6) is rewritten as follows:

$$
\frac{1}{2} \Delta\left(H_{j i} H^{j i}\right)-\nabla_{j}\left(H^{j i} f_{\imath}\right)=\nabla_{k} H_{j i} \nabla^{k} H^{j i}-f_{\imath} f^{i}+\sum_{i<j}\left(c+k_{i} k_{j}\right)\left(k_{i}-k_{j}\right)^{2},
$$

where $f_{\imath}=\nabla_{\imath} f$ and $f^{i}=g^{j i} f_{\jmath}$.

\section{Hypersurfaces with constant scalar curvature.}

We assume in this section that $M$ is a hypersurface with constant scalar curvature $R$ in $\bar{M}$. We shall investigate the sign of the right hand side of (1.7). First of all, we consider the first term and the second term of the right hand side of equation (1.7). By calculating the square of the norm of $f \nabla_{k} H_{j i}-f_{k} H_{j i}$, we get

$$
\left\|f \nabla_{k} H_{j i}-f_{k} H_{j i}\right\|^{2}=f^{2} \nabla_{k} H_{j i} \nabla^{k} H^{j i}-2 f f^{k} H^{j i} \nabla_{k} H_{j i}+f_{k} f^{k} H_{j i} H^{j i} .
$$

From (1.5) it follows that

$$
H_{j i} H^{j i}=c n(n-1)+f^{2}-R,
$$

and, moreover we find

$$
H^{j i} \nabla_{k} H_{j i}=\nabla_{k}\left(H_{j i} H^{j i}\right) / 2=\nabla_{k} f^{2} / 2=f f_{k},
$$

because the scalar curvature $R$ is constant. Eliminating $H_{j i} H^{j i}$ and $H^{j i} \nabla_{k} H_{j i}$ from these equations, we obtain

$$
\left\|f \nabla_{k} H_{j i}-f_{k} H_{j i}\right\|^{2}=f^{2}\left(\nabla_{k} H_{j i} \nabla^{k} H^{j i}-f_{\imath} f^{i}\right)-\{R-c n(n-1)\} f_{\imath} f^{i} .
$$

We now define a domain $D$ in $M$ as follows: $D$ is the set of points $x$ in $M$ such that

$$
\left(\nabla_{k} H_{j i} \nabla^{k} H^{j i}-f_{\imath} f^{i}\right)(x)<0 .
$$


Making use of (2.1), we can prove the following lemma, which gives a sufficient condition for the algebraic sum of the first and the second terms of the right hand side in (1.7) to be non-negative.

LEMMA 2.1. Let $M$ be a hypersurface with constant scalar curvature $R$ in $\bar{M}$. If $R \geqq c n(n-1)$, then the domain $D$ is empty.

Proof. We suppose that $D$ is not empty. By means of the definition of the domain $D$, it follows from (2.1) that

$$
0 \geqq f^{2}\left(\nabla_{k} H_{j i} \nabla^{k} H^{j i}-f_{\imath} f^{i}\right) \geqq\{R-c n(n-1)\} f_{\imath} f^{i} \quad \text { on } \quad D .
$$

Since the scalar curvature $R$ is equal to or greater than $c n(n-1)$, the inequality above shows that $R=c n(n-1)$ or $f_{\imath}$ vanishes identically on $D$. We consider first the case in which the scalar curvature is equal to $c n(n-1)$. The left hand side of (2.1) being equal to or greater than zero, we get

$$
f^{2}\left(\nabla_{k} H_{j i} \nabla^{k} H^{j i}-f_{\imath} f^{i}\right) \geqq 0 .
$$

This means that $f$ vanishes on $D$. Since the domain $D$ is open, we obtain

$$
f_{\imath}=0 \quad \text { on } D \text {. }
$$

This shows that $R=c n(n-1)$ implies that $f_{\imath}=0$ on $D$. When $f_{\imath}=0$ on $D$, the scalar function $\nabla_{k} H_{j i} \nabla^{k} H^{j i}-f_{\imath} f^{i}$ is equal to or greater than zero on $D$. Thus the domain $D$ must be empty, which proves the Lemma.

For each point $x$ in $M$, let $X_{1}, X_{2}, \cdots, X_{n}$ be an orthonormal frame of the tangent space $M_{x}$ such that any $X_{\jmath}$ is an eigenvector of the second fundamental tensor corresponding to an eigenvalue $k_{\jmath}$. Then, by remembering equation (1.1) of Gauss, the sectional curvature $K\left(X_{\imath}, X_{j}\right)$ of the plane section spanned by $X_{\imath}$ and $X_{j}$ is given by

$$
K\left(X_{\imath}, X_{j}\right)=c+k_{i} k_{\jmath} .
$$

Taking account of this relation and remembering the right hand side of equation (1.7), we see that if $M$ is of non-negative curvature and with constant scalar curvature $R \geqq c n(n-1)$, then the right hand side is non-negative. Thus we can prove the following.

LEMMA 2.2. Let $M$ be a compact orientable hypersurface of non-negative curvature and with constant scalar curvature $R$ in $\bar{M}$. If $R \geqq c n(n-1)$, then there exist at most two distinct principal curvatures, say $\lambda$ and $\mu$, such that

$$
c+\lambda \mu=0 .
$$

Proof. Following Lemma 2.1, the assumption $R \geqq c n(n-1)$ implies that the domain $D$ is empty. This shows that 


$$
\nabla_{k} H_{j i} \nabla^{k} H^{j i}-f_{\imath} f^{i} \geqq 0 \quad \text { on } M \text {. }
$$

On the other hand, by virtue of the Green's theorem and equation (1.7), we obtain

$$
\int_{M}\left\{\nabla_{k} H_{j i} \nabla^{k} H^{j i}-f_{\imath} f^{i}+\sum_{i<j}\left(c+k_{i} k_{j}\right)\left(k_{i}-k_{j}\right)^{2}\right\} d M=0,
$$

$d M$ being the volume element of $M$. Thus, $\nabla_{k} H_{j i} \nabla^{k} H^{j i}-f_{\imath} f^{i}$ and $c+k_{i} k_{\jmath}$ being both non-negative, we find

$$
\nabla_{k} H_{j i} \nabla^{k} H^{j i}-f_{\imath} f^{i}=0
$$

and

$$
\left(c+k_{i} k_{j}\right)\left(k_{i}-k_{j}\right)^{2}=0
$$

for any indices $i$ and $j$ at each point in $M$. Equation (2.5) shows that for distinct principal curvatures $k_{i}$ and $k_{\jmath}$ we have

$$
c+k_{i} k_{\jmath}=0 \text {. }
$$

This means that the number of distinct principal curvatures at each point is at most two, and they satisfy (2.3).

We prove now the following main theorem.

THEOREM 2.3. Let $\bar{M}$ be a complete and simply connected Riemannian $(n+1)$ manifold of constant curvature $c$, and let $M$ be a connected compact Riemannian $n$-manifold. Let $\phi$ be an isometric immersion of $M$ into $\bar{M}$. Suppose that $M$ is of non-negative curvature and with constant scalar curvature $R$. If $R \geqq c n(n-1)$, then $(M, \phi)$ is totally umbilic or there exist exactly two distinct and constant principal curvatures.

Proof. Taking account of equations (2.1) and (2.4), and the assumption $R \geqq c n(n-1)$, we see easily that the equation

$$
\{R-c n(n-1)\} f_{\imath} f^{i}=0
$$

holds. We consider the case in which $R$ is different from $c n(n-1)$ and the case in which $R=c n(n-1)$, separately.

In the first case, $f$ is constant. Accordingly, by a theorem of Nomizu and Smyth [9], the assertion of Theorem 2.3 is true.

In the other case, it follows from (1.5) that

$$
f^{2}-H_{j i} H^{j i}=0 \text {. }
$$

Using principal curvatures, we can rewrite this equation as

$$
\sum_{\imath<\jmath} k_{i} k_{\jmath}=0
$$


Also, by the assumption that the sectional curvature is non-negative, we see that the scalar curvature $R$ is non-negative and so is the constant curvature $c$.

Suppose that $c$ is equal to zero, that is to say, the ambient space $\bar{M}$ is an $(n+1)$-dimensional Euclidean space $E^{n+1}$. We get by $(2.6)$ and the assumption that the hypersurface is of non-negative curvature

$$
k_{i} k_{\jmath}=0 \quad \text { for any distinct indices } i \text { and } j .
$$

This implies that the type number $t(x)$ at each point $x$ in $M$ is equal to 0 or 1. Since $M$ is compact, it is seen that there exists a point in $M$ at which all principal curvatures of $M$ are positive or negative. This contradicts the fact that the type number is equal to 0 or 1 . Thus the constant curvature $c$ must be positive.

Next, suppose that there exists a non-umbilic point $p$ in $M$, at which we have two distinct principal curvatures $\lambda(p)$ and $\mu(p)$. Then, by means of (2.3), they satisfy $c+\lambda(p) \mu(p)=0$. Hence one is positive and the other is negative. Under this situation, there exists a maximal connected open set $U$ consisting of non-umbilic points, which contains $p$. At each point in $U,(M, \phi)$ has exactly two distinct principal curvatures with constant multiplicities $k$ and $n-k$, respectively. Then equation (2.6) is equivalent to

$$
\frac{k(k-1)}{2} \lambda^{2}+k(n-k) \lambda \mu+\frac{(n-k)(n-k-1)}{2} \mu^{2}=0
$$

Taking account of (2.3) and (2.7), we see that $\lambda$ and $\mu$ are constant on $U$. This shows that $\lambda$ is different from $\mu$ at the boundary point of $U$ and therefore, by the definition of $U$, the closure $\bar{U}$ of $U$ should be contained in $U$. Thus $U$ is closed. Since $M$ is connected, $U$ is $M$ itself. This completes the proof.

In the case in which the ambient space is Euclidean, we have, as a direct consequence of Theorem 2.3, the following

THEOREM 2.4. Let $M$ be a connected compact Riemannian n-manifold of nonnegative curvature and let $\phi$ be an isometric immersion of $M$ into $E^{n+1}$. If the scalar curvature $R$ of $M$ is constant, then $M$ is isometric to a sphere $S^{n}$ and $\phi$ is an imbedding.

It is seen (for the detail, see [8] or [9]) that model forms of hypersurfaces with constant scalar curvature in a sphere $S^{n+1}(c)$ are given as follows: the great sphere, the small sphere as a totally umbilic hypersurface, and the product space $S^{k}\left(c_{1}\right) \times S^{n-k}\left(c_{2}\right)$, where $1 / c_{1}+1 / c_{2}=1 / c$ and $1 \leqq k \leqq n-1$. By a theorem of Nomizu and Smyth [9], we find

THEOREM 2.5. Let $M$ be a connected compact Riemannian n-manifold of nonnegative curvature and with constant scalar curvature $R$, and let $\phi$ be an isometric immersion of $M$ into $S^{n+1}(c)$. If $R \geqq c n(n-1)$, then $\phi(M)$ is isometric to one of the following spaces: 
(1) the great sphere, and $\phi$ is an imbedding;

(2) the small sphere, and $\phi$ is an imbedding;

(3) $S^{k}\left(c_{1}\right) \times S^{n-k}\left(c_{2}\right)$, where $1 / c_{1}+1 / c_{2}=1 / c$. Except for $k=1$ or $n-1, \phi$ is an imbedding.

The following result is trivial, because of the property of principal curvatures.

Corollary 2.6. Let $M$ be a connected compact Riemannian n-manifold of non-negative curvature and with constant scalar curvature. If, for an isometric immersion $\phi: M \rightarrow S^{n+1}(c), \phi(M)$ is a certain hemi-sphere, open or closed, in $S^{n+1}(c)$, then $M$ is isometric to $S^{n}$ and $\phi$ is an imbedding.

We treat with the case in which the ambient space is a hyperbolic space $H^{n+1}(c)$. As is already known [6], [8], models of hypersurfaces with constant scalar curvature in $H^{n+1}(c)$ are listed up as follows: a sphere $S^{n}$, a hyperbolic space $H^{n}$, a flat space $F^{n}$ as a totally umbilic hypersurface, and $S^{k}\left(c_{1}\right) \times H^{n-k}\left(c_{2}\right)$, where $1 / c_{1}+1 / c_{2}=1 / c$ and $1 \leqq k \leqq n-1$.

Let $M$ be a compact hypersurface of non-negative curvature and with constant scalar curvature immersed in $H^{n+1}(c)$. Then we set

$$
R-c n(n-1)>0 \text {. }
$$

This shows that $M$ satisfies the assumption of Theorem 2.3, and therefore $M$ is totally umbilic or $M$ has exactly two distinct and constant principal curvatures. By a similar method to that used in [6] and [8], it is seen that $M$ is isometric to one of model hypersurfaces stated above. Taking account of the fact that $M$ is compact, we see that $M$ must be isometric to a sphere. Thus we find

TheOREM 2.7. Let $M$ be a connected compact Riemannian n-manifold of nonnegative curvature and with constant scalar curvature, and let $\phi$ be an isometric immersion of $M$ into $H^{n+1}(c)$. Then $M$ is isometric to a sphere and $\phi$ is an imbedding.

\section{Convex hypersurfaces in $E^{n+1}$.}

Let $M$ be a complete hypersurface of non-negative curvature immersed in $E^{n+1}$. Since it is easily seen that any two principal curvatures $k_{i}$ and $k_{\jmath}$ satisfy $k_{i} k_{j} \geqq 0$, we may assume that

$$
k_{1} \geqq k_{2} \geqq \cdots k_{n} \geqq 0
$$

at each point in $M$, without loss of generality. We put

$$
\left(\begin{array}{c}
n \\
j
\end{array}\right) H_{J}=\sum_{i_{1}<\cdots<i_{j}} k_{i_{1}} k_{i_{2}} \cdots k_{i_{j}},
$$

$\left(\begin{array}{c}n \\ j\end{array}\right)$ being binomial coefficients. $H_{\jmath}$ is called the $j$-th mean curvature of $M$. This 
shows that any mean curvature $H_{j}$ is non-negative. We see also that the following relation between the $j$-th and the $(j+1)$-st mean curvatures is true:

$$
H_{1} H_{j}-H_{\jmath+1}=\frac{j !(n-j-1) !}{n n !} \sum_{\substack{i_{1}<i_{2} \\ \imath_{3}<\cdots<i_{j+1}}}\left(k_{i_{1}}-k_{i_{2}}\right)^{2} k_{i_{3}} \cdots k_{i_{\jmath+1}} \text {. }
$$

We now assume that the $s$-th mean curvature $H_{s}$ is constant for a fixed $s$. The following two lemmas concern with this case.

LeMmA 3.1. If there exists a point $p$ such that $H_{i}(p)=0$ for an index $i$ such that $1 \leqq i \leqq s$, then $H_{s}=0$.

Proof. By the definition (3.2) of $H_{i}$, we get

$$
\left(\begin{array}{c}
n \\
i
\end{array}\right) H_{i}=k_{1} k_{2} \cdots k_{i}+\cdots
$$

This implies that $k_{1}(p) k_{2}(p) \cdots k_{i}(p)=0$. It follows from this result and (3.1) that

$$
k_{i}(p)=k_{i+1}(p)=\cdots=k_{n}(p)=0 .
$$

Thus it is easily seen that $H_{s}(p)=0$, from which we have

$$
H_{s}=0 \quad \text { on } M \text {, }
$$

because the $s$-th mean curvature $H_{s}$ is constant.

LEMMA 3.2. If $H_{s}=0$, then the type number $t(x)$ at each point $x$ in $M$ is less than $s$.

Proof. Under the assumption and (3.3) for $j=s$, we see that $H_{s+1}$ is nonpositive. Since, in general, any mean curvature is non-negative, we get

$$
H_{s+1}=0 \quad \text { on } M \text {. }
$$

It follows inductively from (3.3) that

$$
H_{s}=H_{s+1}=\cdots=H_{n}=0 \quad \text { on } M \text {. }
$$

By the definition, the condition $H_{n}=0$ on $M$ is equivalent to $k_{1} k_{2} \cdots k_{n}=0$ on $M$, and therefore $k_{n}$ must vanish identically on $M$. This means that

$$
\left(\begin{array}{c}
n \\
n-1
\end{array}\right) H_{n-1}=k_{1} k_{2} \cdots k_{n-1}=0
$$

and we get $k_{n-1}=0$. Repeating the discussion similar to that used in the above, we obtain

$$
k_{s}=k_{s+1}=\cdots=k_{n}=0 \quad \text { on } \quad M \text {. }
$$


This implies that the assertion of the lemma is true.

Now, we consider first the case $H_{s}=0$. As a direct consequence of Lemma 3.2 , the relative nullity $\nu(x)$ of the immersion $\phi$ of $M$ into $E^{n+1}$ at each point $x$ in $M$ is equal to or greater than $n-s+1$. Thus it follows from a theorem of $P$. Hartman [4] the following is proved:

THEOREM 3.3. Let $M$ be a complete Riemannian n-manifold of non-negative curvature and let $\phi$ be an isometric immersion of $M$ into $E^{n+1}$. If $H_{s}=0$ on $M$, then $\phi(M)$ is $(n-s+1)$-cylindrical and is not $(n-s+2)$-cylindrical.

We consider a hypersurface $M$ imbedded in $E^{n+1}$. A support function is by definition the oriented distance from a fixed point in $E^{n+1}$ to the hyperplane $H_{x}$ in $E^{n+1}$ tangent to $M$ at each point $x$ in $M$. The following theorem is proved by Hsiung [5]: For a compact orientable hypersurface $M$ imbedded in $E^{n+1}$, suppose that a support function is of the same sign. If $H_{j}>0$ for $j=1,2, \cdots, s$ and $H_{s}$ is constant, then $M$ is isometric to $S^{n}$. Let $M$ be a convex hypersurface imbedded in $E^{n+1}$, which is not divided into two parts by $H_{x}$ in $E^{n+1}$ tangent to $M$ at each point $x$ in $M$. Then the second fundamental form is semi-definite and therefore all of principal curvatures are non-positive or non-negative and a support function is of the same sign. Under this situation, since Lemma 3.1 implies that an $i$-th mean curvature $H_{i}$ for any $i=1,2, \cdots, s-1$ is positive provided that $H_{s}>0$, we can prove the following theorem using Lemma 3.2 and the theorem of Hsiung.

THEOREM 3.4. Let $M$ be a compact convex hypersurface imbedded in $E^{n+1}$. If the $s$-th mean curvature $H_{s}$ is constant, then $M$ is isometric to $S^{n}$.

By a theorem of R. Sacksteder [12] and Theorem 3.4, we find

Corollary 3.5. Let $M$ be a compact Riemannian n-manifold of non-negative curvature and let $\phi$ be an isometric immersion of $M$ into $E^{n+1}$. If $H_{s}$ is constant, then $M$ is isometric to $S^{n}$ and $\phi$ is an imbedding.

REMARK. We consider a strictly convex hypersurface $M$ in $E^{n+1}$, that is to say, a hypersurface which is convex and each point $x$ is the only one point lying on the hyperplane $H_{x}$. Then all the principal curvatures are positive or negative. This means, by virtue of the equality (3.3), that the following theorem holds: Let $M$ be a compact strictly convex hypersurface in $E^{n+1}$. If $H_{s}$ is constant, then $M$ is isometric to $S^{n}$. (See Yano [16], p. 86, Theorem 2.2). Theorem 3.4 is a slight generalization of this result.

REMARK. Aleksandrov [1] has proved the following theorem: Let $M$ be $a$ compact Riemannian $n$-manifold and let $\phi$ be an isometric imbedding of $M$ into $S^{n+1}, E^{n+1}$ or $H^{n+1}$. Suppose that $\phi(M)$ is contained in a closed hemi-sphere, provided that the ambient space is a sphere. If a given function $F$ of principal curvatures $k_{1}, k_{2}, \cdots, k_{n}$ of class $C^{1}$ is constant on $M$ and $\partial F / \partial k_{j}>0$ for any index $j$, then $M$ is isometric to $S^{n}$. It is to be regretted that the authors could not 
understand the proof. The condition that the $s$-th mean curvature $H_{s}$ is constant on a compact strictly convex hypersurface in $E^{n+1}$ is satisfied if the assumption of Aleksandrov is satisfied. Though, as is already mentioned, Theorem 3.4 is a slight generalization of a compact strictly convex case, we note that the assumption of convexity is not mentioned in the theorem of Aleksandrov.

REMARK. The following relation between the second mean curvature $H_{2}$ and the scalar curvature $R$ holds:

$$
R=n(n-1)\left(c+H_{2}\right)
$$

This means that Corollary 3.5 is a generalization of Theorem 2.4.

\section{BIBLIOGRAPHY}

[1] Aleksandrov, A. D., Uniqueness theorems for surfaces in the large, I V. I, Amer. Math. Soc. Translations 21 (1962), 341-354; II, ibid., 354-388; III, ibid., 389-403; IV, ibid., 403-411; V, ibid., 412-416.

[2] AMUR, K., Vector forms and integral formulas for hypersurfaces in Euclidean space. J. of Differential Geometry 3 (1969), 111-123.

[ 3 ] Chern, S. S., M. do CARmo, and S. Kobayashi, Minimal submanifolds of a sphere with second fundamental form of constant length. Functional Analysis and Related Fields (Proc. of a conf. in honor of Prof. M. Stone at Univ. of Chicago, 1968), Springer-Verlag (1970), 59-75.

[4] Hartman, P., On the isometric immersions in Euclidean space of manifolds with non-negative sectional curvatures, II. Trans. Amer. Math. Soc. 147 (1970), 529-540.

[5] Hsiung, C. C., Some integral formulas for closed hypersurfaces. Math. Scand. 2 (1954), 286-294.

[6] Lawson Jr., H. B., Local rigidity theorems for minimal hypersurfaces. Ann. of Math. 89 (1969), 187-197.

[7] LiebmanN, H., Über die Verbiegung der geschlossen Flächen positiver Krümmung. Math. Ann. 53 (1900), 91-112.

[8] Mogi, I., and H. Nakagawa, On hypersurfaces with parallel Ricci tensor in a Riemannain manifold of constant curvature. Differential Geometry, in honor of K. Yano, Kinokuniya, Tokyo (1970), 267-279.

[9] Nomizu, K., ANd B. Smyth, A formula of Simons' type and hypersurfaces with constant mean curvature. J. of Differential Geometry 3 (1969), 367-377.

[10] Reilly, R. C., The Gauss map in the study of submanifolds of spheres. Thesis in Univ. of California at Berkeley (1969).

[11] Ryan, P. J., Homogeneity and some curvature conditions for hypersurfaces. Tôhoku Math. J. 21 (1969), 363-388.

[12] Sacksteder, R., On hypersurfaces with non-negative sectional curvatures. Amer. J. Math. 82 (1960), 609-630.

[13] Shahin, J. K., Some integral formulas for closed hypersurfaces in Euclidean space. Proc. Amer. Math. Soc. 19 (1968), 609-613.

[14] Simons, J., Minimal variety in Riemannian manifolds, Ann. of Math. 88 (1968), $62-105$. 
[15] Süss, W., Zur relativen Differentialgeometrie V. Tôhoku Math. J. 30 (1929), 202-209.

[16] YANO, K., Integral formulas in Riemannian geometry. Marcel Dekker, Inc., New York (1970).

[17] YANO, K., AND M. TANI, Integral formulas for closed hypersurfaces. Kōdai Math. Sem. Rep. 21 (1969), 335-349.

Tokyo University of Agriculture and Technology. 